Of 275 patients with DMARDb, 41 admissions for infection in 32 patients $(11.6 \%)$; more than once. 20 (62.5\%), were women, with mean age 69.2 \pm 2.12 years, mean time evolution $24.5 \pm 22.6$ years. Diagnosis was RA: $22(69 \%)$ patients, ankylosing spondylitis: 7 (22\%), psoriatic arthritis: 3 (9\%). Concomitant with DMARDcs in $81 \%$ and $26 \%$ corticosteroid (mean dose: $4.6 \pm 2.6 \mathrm{mg})$. The diagnosis of 41 admitted was: Pneumonia: 9 (41\%), non-pneumonic respiratory infection: 5 (24\%), septic arthritis: 8 $(24 \%)$, urinary infection-pyelonephritis urinary o sepsis: $8(22 \%)$, influenza A: $3(7 \%)$, abscess, gastroenteritis and leishmaniasis in $2(5 \%)$ each, osteomyelitis and varicella in $1(2 \%)$ each.

The DMARDb at admission were: anti-TNF: 34 (83\%) patients (adalimumab: $19 / 46 \%$, etanercept: $7 / 17 \%$, infliximab: $4 / 10 \%$, golimumab and certolizumab: 2/5\% each), tocilizumab: 4 (10\%), abatacept: 2 (5\%).

When comparing patients admitted for infection, patients with DMARDb has higher number of admissions (41 (67\%) vs 20 (33\%), p <0.01), and lower percentage of DMARDcs $(23 / 72 \%)$ vs $16(100 \%)$. $p=0.02)$ and corticosteroids $(14 / 34 \%$ vs $10 / 50 \%)$. $p<0.001)$, at similar doses $(4.7 \pm 2.6$ vs $4.5 \pm 2.6 \mathrm{mg}, \mathrm{p}=0.7)$. The incidence of admission for infection are indicated in table.

Conclusion: 1 . The prevalence of admission due to infection in DMARDb is $9 \%$ and $4 \%$ in DMARDs in monotherapy. 2. Admission predominates in RA, with a long evolution, with biological therapy. 2. The most frequent infections were respiratory origin, followed by sepsis or bacteremia and septic arthritis.

\begin{tabular}{|c|c|c|c|}
\hline & DMARDb & DMARDcs & $\begin{array}{l}\text { DMARDb } \\
\text { +DMARDcs }\end{array}$ \\
\hline $\begin{array}{l}\text { Admission general incidence for } \\
\text { infection }\end{array}$ & $\begin{array}{l}1.92(0.88- \\
3.58)\end{array}$ & $0.95(0.6-1.42)$ & $2.6(1.55-4.05)$ \\
\hline Admission incidence for infection in RA & $\begin{array}{l}4.1(1.47- \\
8.82)\end{array}$ & $\begin{array}{c}1.16(0.72- \\
1.75)\end{array}$ & $2.64(1.37-4.53)$ \\
\hline $\begin{array}{l}\text { Mean time (years) to admission due to } \\
\text { infection } 1\end{array}$ & $\begin{array}{c}7.77(4.17- \\
11.36)\end{array}$ & $\begin{array}{l}19.17(13.83- \\
24.52)\end{array}$ & $6.15(4.06-8.23)$ \\
\hline $\begin{array}{l}\text { Mean time (years) until admission for } \\
\text { infection, in RA }\end{array}$ & $\begin{array}{c}9.05(3.58- \\
14.53)\end{array}$ & $\begin{array}{l}17.95(12.99- \\
22.9)^{2}\end{array}$ & $6.6(3.93-9.26)$ \\
\hline
\end{tabular}

infection, in RA

${ }^{1} p<0.001$

${ }^{2} p<0.001$, DMARDb vs DMARDcs

Acknowledgement: The study was supported with a research grant from the Association for Research in Rheumatology of Marina Baixa (AIRE$\mathrm{MB}$ )

Disclosure of Interests: Jose Rosas Consultant for: Abbvie, Amgen, Bristol, Janssen, Lilly, Merck Sharp \& Dohme, Pfizer, UCB Pharma, Speakers bureau: Abbvie, Amgen, Bristol, Janssen, Lilly, Merck Sharp \& Dohme, Pfizer, UCB Pharma, Ana Pons: None declared, José Alberto GarcíaGómez: None declared, José Miguel Senabre-Gallego: None declared, Gregorio Santos Soler: None declared, Esteban Salas-Heredia: None declared, José Antonio Bernal-Vidal: None declared, Catalina Cano: None declared, Estíbaliz Ivars: None declared, Xavier Barber: None declared DOI: 10.1136/annrheumdis-2019-eular.3841

\section{SAT0471 HIGH-RESOLUTION MELTING CURVE ANALYSIS: A RAPID AND PRAGMATIC APPROACH FOR SCREENING OF MULTIDRUG RESISTANT OSTEOARTICULAR TUBERCULOSIS (OATB)}

Kusum Sharma ${ }^{1}$, Aman Sharma ${ }^{2}$, Mandeep Dhillon ${ }^{3} .{ }^{1}$ Post Graduate Institute of Medical Education and Research, Chandigarh, Medical Microbiology, Chandigarh, India; ${ }^{2}$ Post Graduate Institute of Medical Education and Research, Chandigarh, Internal Medicine, Chandigarh, India; ${ }^{3}$ Post Graduate Institute of Medical Education and Research, Chandigarh, Orthopaedics, Chandigarh, India

Background: Emergence of MDR in extrapulmonary tuberculosis (EPTB) is a major concern in endemic areas. Timely diagnosis and screening of multidrug resistance in patients of Osteoarticular Tuberculosis (OATB) is a challenge.

Objectives: To evaluate the rpoB gene real time PCR (RT PCR) in synovial fluid/pus samples and its comparison with MPB64 and IS6110 RT PCR for diagnosis of OATB.

Methods: Real time PCR using IS6110, MPB64 and rpoB genes was carried out in 150 cases of OATB and in100 non TB control group. Out of these, 20 OATB cases were culture confirmed and 130 were clinically suspected cases. Phenotypic susceptibility testing for culture isolates was performed by standard proportion method.DNA extracted from samples of the culture confirmed cases and the suspected OATB cases confirmed by RT PCR were subjected to rpoB and katG HRM analysis for screening of MDR.
Results: The sensitivity of rpoB RT PCR, MPB64 RT PCR and IS6110 RT PCR was $86.5 \%, 86.5 \%$ and $76.5 \%$ respectively. All RT PCR were negative in the control group thus the specificity was $100 \%$. HRM analysis detected rifampicin resistance in 10 cases of which, $8(80 \%)$ were MDR while $2(20 \%)$ had isolated rifampicin resistance. Of the 13 cases of isoniazid resistance detected by HRM analysis, $8(61.5 \%)$ were MDR while $5(38.46 \%)$ were isolated isoniazid resistant. HRM analysis detected an additional 4 MDR cases directly from the samples which were negative by culture. Subsequently, results of HRM analysis were confirmed by rpoB sequencing and mutation were observed at codon $531(60 \%)$; 533 $(16 \%) ; 516(12 \%)$ and $526(12 \%)$. katG sequencing revealed mutation at codon $315(100 \%)$. There was $100 \%$ concordance in the results of phenotypic DST, sequencing results and HRM analysis.

Conclusion: rpoB and katG HRM analysis is a promising method in reliable and rapid screening of drug resistance in OATB cases in 90 minutes.

Disclosure of Interests: None declared

DOI: 10.1136/annrheumdis-2019-eular.6941

\section{SAT0472 THE CONTRIBUTION OF MRI IN THE DIAGNOSIS OF INFECTIOUS SPONDYLODISCITIS}

Rawdha Tekaya, Khaoula Zouaoui, Aicha Ben Tekaya, Lobna Ben Ammar, Olfa Saidane, Ines Mahmoud, Leila Abdelmoula. Charles Nicolle Hospital, Tunis, Tunisia

Background: Infectious spondylodiscitis is a diagnostic and therapeutic emergency, and if there is any doubt, an MRI should be performed to support the diagnosis.

Objectives: The aim of our study is to highlight the role of MRI in the diagnosis of infectious spondylodiscitis.

Methods: This is a 20-year retrospective study (1999-2019) performed at a rheumatology department, collecting cases of infectious spondylodiscitis We have identified the epidemiological, clinical, and radiological characteristics.

Results: Our study included 106 patients, 57 men and 49 women. The average age was $55[16 ; 86]$. Mean disease duration averaged 4.54 months [0.23; 24]. All patients had spinal pain: lumbar in $65.09 \%$ of cases, dorsal in $17 \%$ of cases and cervical in $6 \%$ of cases. Standard Xrays showed abnormalities suggestive of spondylodiscitis in $88.67 \%$ of cases. Magnetic resonance imaging was then performed in $76.41 \%$ patients and was pathological in all cases. The abnormalities found were paravertebral abscess in $23.58 \%$ of cases, epiduritis in $10.37 \%$ of cases and association of abscess and epiduritis in $32.07 \%$ of cases. The rest of the abnormalities noted were vertebral osteolysis $(8.49 \%)$, spinal cord compression $(9.43 \%)$ abnormalities of the intervertebral disc with narrowing and/or signal modification (4\%). The abnormalities were monofocal in $82.07 \%$ of cases and bifocal in $13.2 \%$ of cases. $78.57 \%$ of bifocal lesions were objectified in tuberculous spondylodiscitis. $82.07 \%$ of the spinal injuries were single-stage and $15.09 \%$ were bi-staged. $75 \%$ of these were of tuberculous origin. There were $2.83 \%$ of spondylodiscites up to 3 stages and all were tuberculous.

Conclusion: MRI is a great diagnostic aid during infectious spondylodiscitis. It also allows to orient towards the etiological diagnosis.

Disclosure of Interests: None declared

DOI: 10.1136/annrheumdis-2019-eular.6280

\section{SAT0473 MUSCULOSKELETAL STIFFNESS IN CHIKUNGUNYA DISEASE: DISTINCT FROM PAIN AND RELEVANT TO QUALITY OF LIFE}

Hugh Watson ${ }^{1}$, Sarah Tritsch ${ }^{2}$, Liliana Encinales ${ }^{3}$, Andres Cadena ${ }^{4}$, Carlos Cure ${ }^{5}$, Alexandra Porras ${ }^{6}$, Alejandro Rico Mendoza ${ }^{6}$, Aileen Chang ${ }^{2} .{ }^{1}$ Aarhus University, Department of Clinical Medicine, Aarhus, Denmark; ${ }^{2}$ The George Washington University, School of Medicine and Health Sciences, Washington, United States of America; ${ }^{3}$ Allied Research Society LLC, Barranquilla, Atlantico, Colombia; ${ }^{4}$ Clinical de La Costa Ltda, Barranquilla, Atlantico, Colombia; ${ }^{5}$ Biomelab, Barranquilla, Atlantico, Colombia; ${ }^{6}$ Universidad El Bosque, Grupo de Medicina Communitaria y Salud Colectiva, Bogota, Colombia

Background: Musculoskeletal stiffness is reported to be frequent following chikungunya infection and can persist for many months after infection. However, stiffness severity and its impact is not well characterised in this disease. A stiffness patient reported outcome instrument has been developed for use in rheumatoid arthritis.

Objectives: Our objective was to assess the use of this questionnaire and importance of musculoskeletal stiffness in a cohort of chikungunya 
patients with chronic joint symptoms in the Atlántico Department of Colombia.

Methods: Sixty-seven patients with chronic arthralgia and 15 patients without arthralgia were followed up a mean of 40 months after chikungunya infection. The patients came from a larger cohort of 500 patients previously followed up 20 months after infection. Those consenting to a 40month in-person follow-up were included here. Tender joint counts, a pain intensity visual analogue scale (VAS), Health Assessment QuestionnaireDisability Index (HAQ-DI) and the EuroQol overall health VAS (EQ-VAS) were completed. A 21-item musculoskeletal stiffness questionnaire (MSQ) was completed and summarized as percentage scores for overall stiffness and its components: stiffness severity, physical impact and psychosocial impact.

Results: The 82 patients (12 male and 70 female) had a mean age 51 \pm 14 years. Forty-two out of sixty-seven patients with arthralgia and $3 / 15$ patients without arthralgia reported musculoskeletal stiffness. Stiffness in those patients had a median severity of $28 \%$ (IQR $0-42$ ). An impact of their stiffness on physical activities was reported by $39 / 45$ patients (87\%) and psychosocial impact by $32 / 45$ patients (71\%). Overall MSQ score was a median of $16 \%$ (IQR 0-34). Mean tender joint count in patients reporting arthralgia was $6.2 \pm 7.1$, mean pain intensity $65 \pm 20$ out of 100 , mean $\mathrm{HAQ}-\mathrm{DI}=0.54 \pm 0.52$, and a mean $\mathrm{EQ}-\mathrm{VAS}=68 \pm 62$ out of 100 .

Overall stiffness scores were poorly correlated with tender joint counts $\left(r^{2}=0.17\right)$ and pain intensity $\left(r^{2}=0.22\right)$. Stiffness scores were more strongly associated with the HAQ-DI $\left(r^{2}=0.52\right)$ and EQ-VAS overall health VAS scores $\left(r^{2}=0.46\right)$, whereas tender joint counts were not: $r^{2}=0.22$ for $H A Q-$ DI and $r^{2}=0.21$ for EQ-VAS.

Conclusion: Musculoskeletal stiffness following chikungunya infection is distinct from the persistent arthralgia usually reported. It does not necessarily occur in the same patients and is poorly correlated with joint pain severity. Stiffness, as measured by this questionnaire, may be more strongly associated than arthralgia with overall health and disability indices in patients with chikungunya disease. The MSQ is a potentially useful instrument for assessing symptoms in chronic chikungunya disease. Disclosure of Interests: Hugh Watson Shareholder of: Sanofi, Employee of: Sanofi, Sarah Tritsch: None declared, Liliana Encinales: None declared, Andres Cadena: None declared, Carlos Cure: None declared, Alexandra Porras: None declared, Alejandro Rico Mendoza: None declared, Aileen Chang: None declared

DOI: 10.1136/annrheumdis-2019-eular.4048

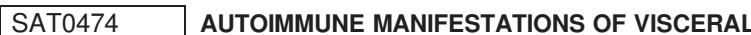 LEISHMANIASIS IN CHINESE PATIENTS}

Xixi Chen, Qiao Zhou, Rui Wu, Jia Cheng, Jian Liu, Bin Zhou, LI Long. Sichuan Provincial People's Hospital, Rheumatology, Chengdu, China

Background: Visceral leishmaniasis (VL), caused by the protozoan Leishmania species, is the most severe form of leishmaniasis. The parasite migrates to the internal organs such as the liver, spleen, and bone marrow, and if left untreated, will almost always cause the host to die [1] Due to polyclonal B cells activation, VL may present signs and symptoms resembling those of rheumatic diseases, especially systemic lupus erythematosus (SLE) [2-4]. This can sometimes lead to mis-diagnosis and treatment of corticosteroids.

Objectives: To analyze 27 Chinese VL patients retrospectively and focused on their autoimmune findings that could help clinicians to differentiate VL from SLE.

Methods: 27 in-hospitalized VL patients in our hospital from 2006 to 2015 were analyzed. VL was diagnosed by the presence of high titers of anti-leishmania antibodies in the blood or by demonstration of intracellular parasites on bone marrow aspiration. All patients were negative for hepatitis B, hepatitis $\mathrm{C}$ and human immunodeficiency viruses. Autoimmune antibodies were detected. Levels of serum globulins, complement C3, C4 and RF were evaluated. Coagulation parameters, including prothrombin time (PT) and activated partial thromboplastin time (APTT) were also tested.

Results: The basic characteristics and laboratory findings of the 27 patients were summarized in Table 1. All patients had hepatosplenomegaly. 38.9\% had positive ANA, but the titer was no more than 1:320. None of the patients had positive anti-Sm antibody and only 1 had antidsDNA antibody. $89.5 \%$ had increased IgG level and none had decreased $\mathrm{C} 3$ or C4.

All patients were cured with sodium stibogluconate, and ANA titers decreased after treatment.

Conclusion: VL may mimic symptoms of SLE due to polyclonal activation of $B$ cells. However, hepatosplenomegaly is a common symptom seen in
VL but not so common in SLE. Furthermore, VL patients don't have typical autoantibodies of SLE, their ANA titer are normal to low and they tend to have normal complements, which could help clinicians to differen tiate these two diseases.

Table 1. Basic characteristics and clinical manifestations of VL patients

\begin{tabular}{lc}
\hline Sex (Femal/Male) & $4 / 23$ \\
\hline Age (median, range) & $37(9-59)$ \\
Disease duration (months) (median, range) & $1(0.17-24)$ \\
Anti- leishmania antibodies positive & 27 \\
Parasites positive in bone marrow & 24 \\
Fever & 25 \\
Hepatosplenomegaly & 27 \\
Weight loss & 14 \\
Anemia & 26 \\
Leucocytopenia & 23 \\
Thrombocytopenia & 20 \\
Prolonged APTT & $12 / 21$ \\
Prolonged PT & $12 / 21$ \\
Positive ANA & $7 / 18$ \\
\hline
\end{tabular}

\section{REFERENCES}

[1] Martins-Melo FR, Lima Mda S, Ramos ANAN Jr., and, et al. Mortality and case fatality due to visceral leishmaniasis in Brazil: a nationwide analysis of epidemiology, trends and s patial patterns. PLoSOne. 2014; 9: e93770.

[2] Ozlem Guzel Tunccan, Abdurrahman Tufan, Gülçin Telli, et al. Visceral Leishmaniasis Mimicking Autoimmune Hepatitis, Primary Biliary Cirrhosis, and Systemic Lupus Erythematosus Overlap. Korean J Parasitol. 2012; 50 (2): $133-6$

[3] Voulgarelis M, Voulgari PV, Serelis J, et al. Visceral leishmaniasis mimick ing systemic lupus erythematosus. J Clin Rheumatol. 2010;16(4): 203-4.

[4] Drosos AA, Skopouli FN. Visceral leishmaniasis resembling systemic lupus erythematosus. Clin Rheumatol. 2003; 22(6):452-5.

Acknowledgement: Thanks to my colleagues who help me in writing the paper and analyzing all the data.

Disclosure of Interests: None declared

DOI: 10.1136/annrheumdis-2019-eular.1493

\section{SAT0475 ECHINOCOCCOSIS AND AUTO-IMMUNE INFLAMMATORY ARTHRITIS; REPORT OF 8 CASES AND REVIEW OF THE LITERATURE}

Pascal Zufferey ${ }^{1}$, Zambaz Camille ${ }^{1}$, Rudiger Muller ${ }^{2}$, Boillat-Blanco Noémie ${ }^{3}$ Thomas Huegle ${ }^{1}$, Hasler Paul ${ }^{4}$, Peter Villiger ${ }^{5} .{ }^{1} R H U$, DAL/CHUV, Lausanne, Switzerland; ${ }^{2}$ rheumatology, Medizinische Universitätsklinik kantonspital, Aarau, Switzerland; ${ }^{3}$ infectiology, Medecine/CHUV, Lausanne, Switzerland; ${ }^{4}$ rheumatology, Medizinische universitästklinik kantonspital, Aarau, Switzerland; ${ }^{5}$ rheumatology, Immuno and allergology, University hospital of Bern, Bern, Switzerland

Background: The prevalence of Echinococcosis in Western Europe and Switzerland has been rising in the past 20 years: $>30$ cases per yea have been reported since 2005 (1). A few case reports (2) and some experimental animal studies (3) have suggested that the immunosuppression linked to inflammatory arthritis and their treatments could favor the development and the growth of parasitic lesions. On the other hand, a recent French study (4) has a shown that the prevalence of Echinococcosis in arthritis patients was close to the normal population. The characteristics of the parasitic disease were quite similar whether inflammatory arthritis was present or not

Objectives: To evaluate the yearly recent incidence of this disease among patients with inflammatory arthritis in Switzerland and to search

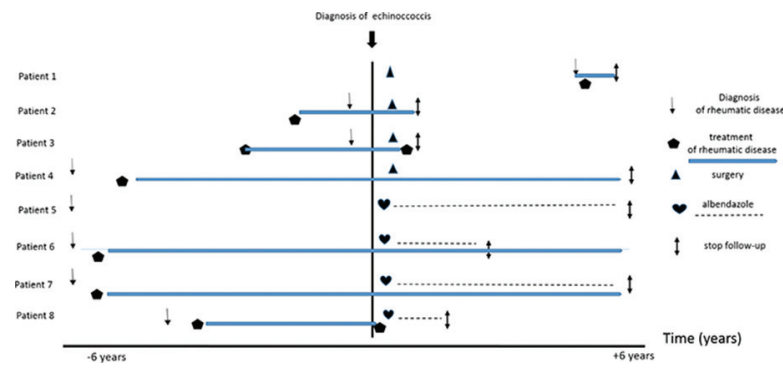

